\title{
Boundary lubrication: Dynamics of squeeze-out
}

\author{
S. Zilberman, ${ }^{1}$ B. N. J. Persson, ${ }^{2}$ A. Nitzan, ${ }^{1}$ F. Mugele,${ }^{3}$ and M. Salmeron ${ }^{3}$ \\ ${ }^{1}$ School of Chemistry, Tel Aviv University, Tel-Aviv 69978, Israel \\ ${ }^{2}$ Institut für Festkorperforschung, Forschungszentrum Jülich, D-52425 Jülich, Germany \\ ${ }^{3}$ Material Sciences Division, Lawrence Berkeley National Laboratory, University of California, Berkeley, California 94720
}

(Received 8 January 2001; published 17 April 2001)

\begin{abstract}
The dynamics of the expulsion of the last liquid monolayer of molecules confined between two surfaces (measured recently for the first time) has been analyzed by solving the two-dimensional Navier-Stokes equation combined with kinetic Monte Carlo simulations. Instabilities in the boundary line of the expelled film were observed. We show that the instabilities produce a rough boundary for all length scales above a critical value and a smooth boundary for shorter lengths. The squeezing out of all but a few trapped islands of liquid is shown to be the result of the pressure gradient in the contact area.
\end{abstract}

DOI: 10.1103/PhysRevE.63.055103

PACS number(s): $81.40 . P q$

Sliding friction is one of the oldest problems in physics, and has undoubtedly a huge practical importance [1-3]. In recent years, the ability to produce durable low-friction surfaces and lubricants has become an important factor in the miniaturization of moving components in technologically advanced devices. For such applications, the interest is focused on the stability under pressure of thin lubricant films, since the complete squeeze-out of the lubricant from an interface may give rise to cold-welded junctions, resulting in high friction and catastrophically large wear.

In this Rapid Communication we investigate the late stages of the approach of two elastic solids limited by two curved surfaces, wetted by a lubricant film of microscopic thickness. Under these conditions, the behavior of the lubricant is mainly determined by its interaction with the solids that induce layering in the perpendicular direction [4-11]. The thinning of the lubrication film occurs stepwise, by expulsion of individual layers [12-15].

The dynamics of the layering transition has been studied with the Surface Forces Apparatus by imaging the gap region in two dimensions [16]. The experiment was performed with a chain alcohol $\mathrm{C}_{11} \mathrm{H}_{23} \mathrm{OH}$ molecule, where the amount of liquid expelled in the layering transitions during slow approach experiments, corresponds to a bilayer of molecules with the OH-groups pointing towards each other [17]. The mica surfaces are covered by strongly bound monolayers of $\mathrm{C}_{11} \mathrm{H}_{23} \mathrm{OH}$ (via the OH-group), that cannot be removed by squeezing, leading effectively to a $\mathrm{CH}_{3}$-terminated substrate for any additional material inside the gap [18]. These coated surfaces are very inert, and the additional alcohol does not wet the surfaces. Shear experiments showed that the static friction force remains zero (and no stick-slip is observed) up to and including the last alcohol layer, indicating that this layer $(n=1)$ is in a $2 \mathrm{D}$-liquid-like state. This is further supported by viscosity measurements, by studying the damping of mica oscillations [18], which shows liquid-like behavior down to the last expelled layer. Once this layer is expelled (corresponding to the $n=0$ situation), the contact between the $\mathrm{CH}_{3}$ terminated films strongly bound to the mica leads to solid-like friction, with nonzero static friction force, and stick-slip during sliding.
The dynamics of the layering transition separates into two phases. In the first phase, the system is trapped in a metastable state at the initial film thickness, i.e., one layer of alcohol molecules between the substrate-bound monolayers. Thermal fluctuations of the two-dimensional density in this layer eventually lead to the formation of a hole with a radius that exceeds the critical radius. Once the nucleus is formed the growth phase begins, and the rest of the layer is quickly expelled. A snapshot picture during squeeze-out is shown in Fig. 1 (see also Ref. [16]).

In this paper we consider the dynamics of expulsion for 2D-liquid-like films. We focus on the evolution of the boundary line separating the $n=1$ and $n=0$ regions during the layering transition $n=1 \rightarrow 0$, when the nucleation of the layering transition occurs off-center. Since the lubrication film is assumed to be in a 2D-liquid-like state, the basic equations of motion for the lubrication film are the continuity equation and the (generalized) Navier-Stokes equation for the 2D-velocity field $\mathbf{v}(\mathbf{x}, t)$ (we assume an incompressible 2D fluid) $[1,12]$,

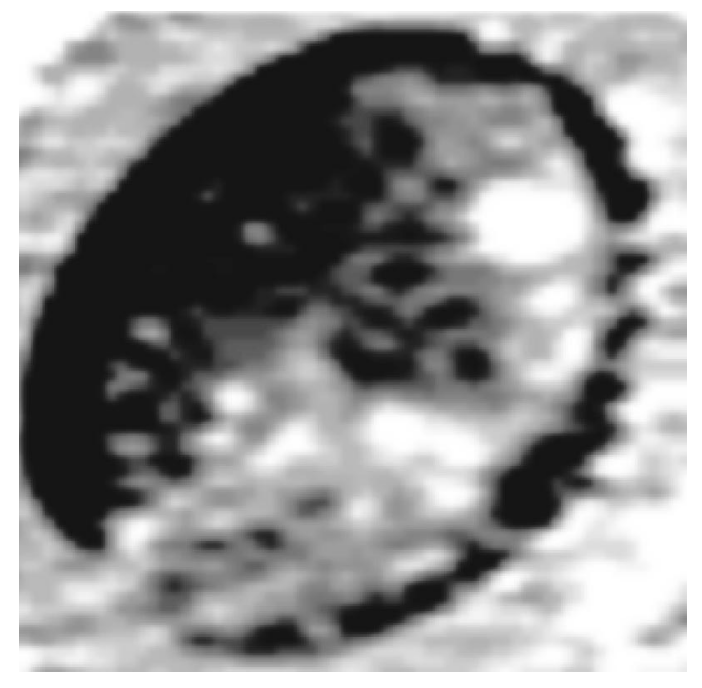

FIG. 1. Snapshot during squeeze out $n=1 \rightarrow 0$ for $\mathrm{C}_{11} \mathrm{H}_{23} \mathrm{OH}$ between mica surfaces. Black area corresponds to $n=0$, and grey to $n=1$. The brightest areas correspond to the buildup of trapped pockets (see Ref. [16]). 


$$
\begin{gathered}
\nabla \cdot \mathbf{v}=0 \\
\frac{\partial \mathbf{v}}{\partial t}+\mathbf{v} \cdot \nabla \mathbf{v}=-\frac{1}{m n_{\mathrm{a}}} \nabla p+\nu \nabla^{2} \mathbf{v}-\bar{\eta} \mathbf{v}
\end{gathered}
$$

where $p$ is the 2D-pressure, $\nu$ the 2D-kinematic viscosity, and $m n_{\mathrm{a}}$ the mass density. The last term in Eq. (2) describes the "drag-force" from the substrate acting on the fluid. It is possible to show by dimensional arguments that, to a good approximation, one can neglect the nonlinear and the viscosity terms in Eq. (2), and assume that the velocity field changes so slowly that the time derivative term can be neglected. Thus,

$$
\nabla p+m n_{a} \bar{\eta} \mathbf{v}=0
$$

From this equation it follows that

$$
\mathbf{v}=\nabla \phi
$$

where

$$
\phi=-p / m n_{a} \bar{\eta}
$$

The continuity equation (1) gives

$$
\nabla^{2} \phi=0 \text {. }
$$

Now, from Eq. (6), we see that the velocity potential can be interpreted as an electrostatic potential. Furthermore, while the 2D-pressure $p$ is constant at the (outer) boundary $r=R$ of the contact area, at the inner boundary towards the $n=0$ area, it depends linearly on the perpendicular (3D) pressure $P(r)(0<r<R)$ via the relation $p_{1}(r)=p_{0}+P(r) a$, where $R$ is the radius of the contact area, $p_{0}$ the spreading pressure, and $a$ the thickness of the monolayer (see Ref. [12]). From Hertz contact theory

$$
P(r)=\frac{3}{2} P_{0}\left(1-\frac{r^{2}}{R^{2}}\right)^{1 / 2}
$$

Thus, the problem of finding $\phi$ is mathematically equivalent to finding the electrostatic potential between two cylinders at different potentials, $\phi_{0}=-p_{0} / m n_{a} \bar{\eta}$ and $\phi_{1}(r)=$ $-p_{1}(r) / m n_{a} \bar{\eta}$, where the outer cylinder has a circular shape (radius $R$ ), and the inner cylinder an unknown (time dependent) shape to be determined. Except for the different boundary conditions, this situation is mathematically very similar to viscous fingering, where the analogy to electrostatics has already been pointed out [see, e.g., Ref. [13(b)]].

It is easy to show that the time evolution of the boundary line is unstable with respect to small perturbations. Let us first consider a perfectly smooth circular boundary line centered at the center of the contact area. For a perfect system (no defects or fluctuations), by symmetry such a boundary line would propagate in a symmetric (circular) way until it reaches the outer boundary of the contact area. Now assume that, due to a fluctuation, a small protrusion is formed on the boundary line, which will locally decrease the distance to the outer boundary line $r=R$. By analogy to electrostatics, this will give rise to an enhanced "draining" velocity of the fluid at the protrusion, so that the boundary line at the protrusion will move faster towards the periphery than in the other regions. This argument is valid for protrusions of any size, and it follows that, within the model discussed above, the boundary line will be rough at all length scales. However, when the free energy (per unit length) $\Gamma$ (line tension) of the boundary line is taken into account (it was neglected above), the boundary line will be smooth on all length scales below some critical cut-off length $\lambda_{c}$, while it will be rough on longer length scales, where $[13,19]$

$$
\lambda_{\mathrm{c}}=2 \pi\left(\Gamma / m n_{a} \bar{\eta} v_{0}\right)^{1 / 2},
$$

where $v_{0}$ is the velocity of the boundary line. The linetension $\Gamma$ has a contribution from unsaturated bonds at the boundary line, and another much larger contribution from the energy stored in the elastic deformation field in the confining solids in the vicinity of the boundary line [13]. Under the experimental conditions in Ref. [16], Eq. (8) predicts $\lambda_{\mathrm{c}}$ $\sim 5 \mu \mathrm{m}$, which equals $1 / 10$ of the diameter of the contact area. The experimental boundary line for $\mathrm{C}_{11} \mathrm{H}_{23} \mathrm{OH}$ is indeed rough at this length scale (see Fig. 1), while it is smooth on shorter length scales. Based on this result one may also argue that the linear size of the trapped islands should be of order $\lambda_{c}$ (or larger), which again agrees with the observations.

We have performed kinetic Monte Carlo (MC) simulations [based on Eqs. (1)-(7)] to study the squeeze-out process in detail. The basic reasoning behind the $\mathrm{MC}$ modeling of the system is quite straightforward. At each MC step, one solves the Laplace equation on a two-dimensional cell centered grid, and the interface line between fluid and squeezed areas has to be moved in a manner that follows flow lines. This is followed by a line relaxation move that imposes line tension on the moving interface.

We have used a volume discretization scheme [20] combined with a successive over relaxation iterative procedure for solving the Laplace equation [21]. Boundary conditions at the periphery were set to zero, while at the interface between squeezed and fluid areas the boundary conditions were position-dependent, determined from the Hertz expression as described above.

Having solved the Laplace equation one can associate with each interface cell $i$ (both fluid and squeezed ones) a velocity parameter $v_{i}$ which represents the sum over all local velocity contributions that "move" the interface line into cell $i$. Since $v_{i}$ represent the flow velocity of a fluid cell into a squeezed cell, or in the opposite direction, we choose the probability for transforming the state of cell $i$ from squeezed $\rightarrow$ fluid or fluid $\rightarrow$ squeezed, as

$$
P_{V}(i)=\frac{v_{i}}{\max \left\{v_{i}\right\}} .
$$

Thus, the probability to accept a tentative MC move is proportional to $v_{i}$, as it should be. Note, however, that the time scale is nonlinearly related to the number of MC steps (since 


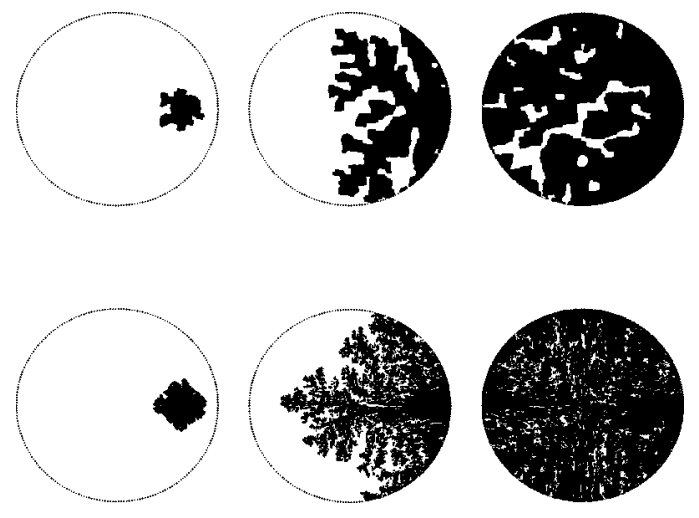

FIG. 2. Snapshots of the layering transition for Hertz contact pressure and with the line tension included (top), and with zero line tension (bottom)

the normalization is different at each MC step); in other words, the time of a single MC step $\Delta t \propto 1 / \max \left\{v_{i}\right\}$.

To account for the line-tension effect we have chosen a simple mechanism of one-dimensional line diffusion of squeezed patches at the interface line. Each line relaxation step was composed of $M$ micro exchange steps, where $M$ is the number of squeezed cells along the interface line. At each microstep, a squeezed interface patch and a target neighboring fluid patch were chosen at random for an exchange process. The probability for accepting the exchange was

$$
P_{l}(E)= \begin{cases}q & \Delta E \leqslant 0 \\ q \mathrm{e}^{-\beta \Delta E} & \Delta E>0,\end{cases}
$$

where $q=\min \left(1, \bar{\eta}_{\mathrm{t}} / \bar{\eta}_{\mathrm{s}}\right)$, where $\bar{\eta}_{\mathrm{t}}$ and $\bar{\eta}_{\mathrm{s}}$ are the sliding friction at the source and target cells, respectively.

In Eq. (10), $\Delta E=\Gamma \Delta l$ is the change in line free energy, and $\Delta l$ is the change in the length of the interface line. For every MC step we usually had a few tens of line relaxation steps. An alternative way to introduce the line tension is via the curvature of the boundary line. This approach is harder to implement, but both treatments should give the same result if enough line boundary diffusion moves are allowed for, so that the boundary line is close to thermal equilibrium at all stages during squeeze-out.

We focus on the dynamics of the layering transition $n$ $=1 \rightarrow 0$. Figure 2 (top) shows snapshot pictures of the layering transition for a Hertzian contact pressure and with realistic line tension. Note that the boundary line is rough for all length scales (fractal) above a lower cut-off length determined by the line tension. Figure 2 (bottom) shows snapshot pictures of the layering transition when the line tension $\Gamma=0$. In this case a fractal pattern occurs for all length scales above the low-distance cut-off length, given by the mesh size. This behavior is in sharp contrast with the experimental data, showing the fundamental importance of the line tension for a correct description of the squeeze-out process.

During squeeze-out (Fig. 2 top) the local curvature of the boundary line between the $n=1$ and $n=0$ regions becomes negative in some areas. Some of these areas eventually detach from the boundary and leave behind pockets of $n=1$

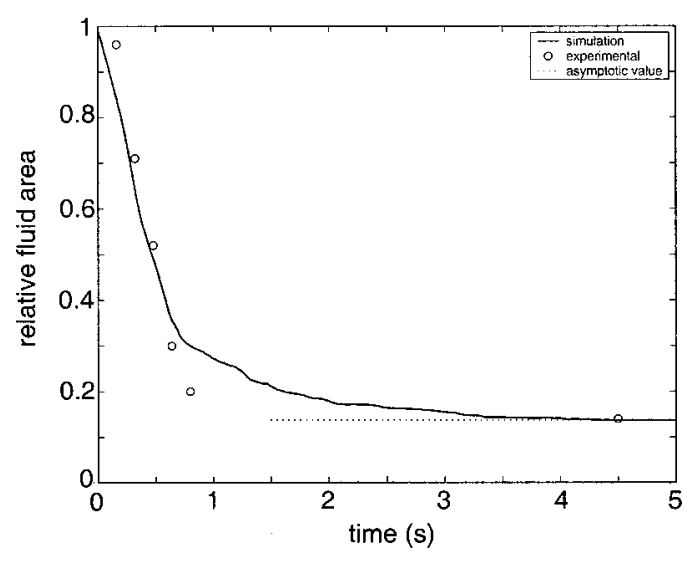

FIG. 3. Fractional area occupied by the fluid as a function of time with the initial position of the squeeze nucleus at $r=0.7 R$. Solid line, theory; circles, experiment.

layer trapped material in the final $n=0$ state. We note that unless an island is centered in the center of the contact area, there will be a net tangential force acting on the island due to the spatial variation in the normal stress from a maximum at the center to zero at the periphery of the contact area. Thus, without defects (pinning centers), the pockets move towards the edge as a whole. There they form little necks through which liquid is squeezed out. This is exactly what is observed in the experiments; see Fig. 1 and Ref. [16]. However, in the experiments some islands are also found to exhibit a negligible (undetectable) drift towards the periphery, indicating that there may be some kind of pinning, even for the liquidlike layers used in the experiments. In order to simulate pinning we have introduced small, high friction areas, where $\bar{\eta}$ was taken to be $10^{4}$ times higher than in the remaining area. This produces pinning of the fluid in these areas, resulting in a finite amount of trapped liquid even for very large times. The solid line in Fig. 3 shows the fractional area occupied by the fluid as a function of time for an initial position of the nucleus at $r=0.7 R$, and with pinning areas corresponding to about $13 \%$ of trapped fluid remaining for longer times. The circles are the experimental results. The

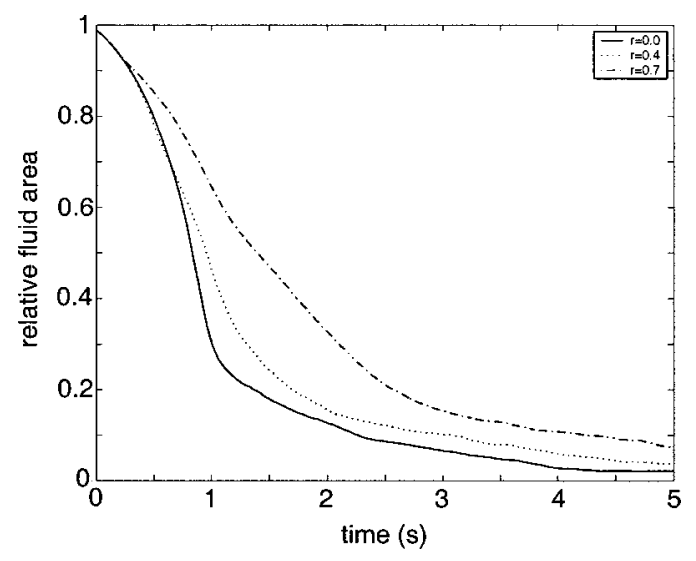

FIG. 4. Fractional area occupied by the fluid as a function of time with the initial position of the squeeze nucleus at the center (solid line), at $r=0.4$ (dotted line), and for $r=0.7 R$ (dash-dotted line). Without pinning areas. 


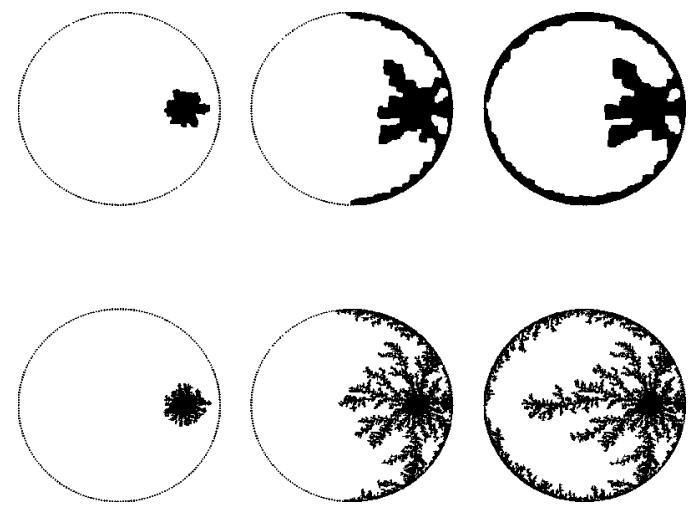

FIG. 5. Snapshots of the layering transition for constant contact pressure and with the line tension included (top), and with zero line tension (bottom).

factor relating the MC time to the real time has been chosen in order to make the agreement with the experimental data as good as possible.

Figure 4 shows the fractional area occupied by the fluid as a function of time for an initial position of the nucleus at the center (solid line), at $r=0.4 R$ (dotted line), and at $r=0.7 R$ (dash-dotted line). Note that one can distinguish between two phases of squeeze-out: an initial "fast" phase up to a time $t \approx 1 \mathrm{~s}$, and a slower phase where the fluid islands are squeezed out. These latter processes take a long time because islands originally located close to the center of the contact area experience a very weak net lateral force.

Figure 5 shows the same as Fig. 2, but now with a constant contact pressure. Note that the fast propagation along the periphery of the contact area causes trapping of a huge fluid island. When a Hertzian contact pressure is assumed, the increase in the squeeze-out speed close to the periphery is much smaller (since $P \rightarrow 0$ as $r \rightarrow R$ ), which makes it possible to squeeze out much more fluid from the interior of the contact area, resulting in much smaller "trapped" islands, in qualitative agreement with experiment. We also note that in Fig. 5 the squeeze-out process stops when the drained area encircles the trapped island. At this point there is no pressure difference across the fluid and the dynamics stops. This is in sharp contrast to the Hertzian contact pressure case, where a squeeze-out force acts radially on any island of "trapped" fluid. Thus, in the latter case it is necessary to introduce pinning centers in order for fluid to remain trapped for large times, as observed in the experiments.

To summarize, a lubrication fluid confined between two approaching surfaces form, in the limit of thin interfaces, well defined layers of molecular thickness, whose number decreases in discontinuous steps with increasing applied pressure. We have studied the dynamics of the squeeze-out by solving the $2 \mathrm{D}$-Navier Stokes equations with an interfacial friction term, and found the results to be in good agreement with the experimental data. We note that it is very important to use a variable (Hertzian) contact pressure profile and to include the line tension: if $\Gamma=0$ or if the contact pressure is assumed to be constant rather than Hertzian, the computer simulations disagree qualitatively with the experiment.

We thank E. Brener and P. Graf for useful discussions. B.P. and A.N. thank BMBF for a grant related to the German-Israeli Project Cooperation "Novel Tribological Strategies from the Nano-to Meso-Scales.', B.P. thanks Pirelli for a grant related to "Physical principles of rubber friction and application to tires.' 'F.M. acknowledges the Alexander von Humbolt foundation, Bonn (Germany). M.S. acknowledges support from the U.S. Department of Energy under Contract No. DE-AC03-76SF00098.
[1] B.N.J. Persson, Sliding Friction: Physical Principles and Applications, 2nd ed. (Springer, Heidelberg, 2000).

[2] B.N.J. Persson, Surf. Sci. Rep. 33, 83 (1999).

[3] J. Krim, Sci. Am. 275, 74 (1996).

[4] J.N. Israelachvili, Intermolecular and Surface Forces (Academic Press, London, 1995).

[5] M.L. Gee, P.M. McGuiggan, and J.N. Israelachvili, J. Chem. Phys. 93, 1895 (1990).

[6] J. Gao et al., Phys. Rev. Lett. 79, 705 (1997).

[7] H. Tamura et al., Langmuir 15, 7816 (1999).

[8] F. Mugele and M. Salmeron, J. Chem. Phys. 114, 1831 (2000).

[9] L. Demirel and S. Granick, J. Chem. Phys. 106, 6889 (1998).

[10] J. Klein et al., Science 269, 816 (1995).

[11] E. Kumacheva et al., J. Chem. Phys. 108, 7010 (1998).

[12] B.N.J. Persson and E. Tosatti, Phys. Rev. B 50, 5590 (1994).

[13] (a) B.N.J. Persson, Chem. Phys. Lett. 324, 231 (2000). (b) See also Fractals and Disordered Systems, edited by A. Bunde and
S. Havlin (Springer, Berlin, 1991); (c) J.S. Langer, Phys. Rev. A 36, 3350 (1987); E. Brener, Phys. Rev. Lett. 71, 3653 (1993).

[14] B.N.J. Persson and P. Ballone, Solid State Commun. 115, 599 (2000).

[15] B.N.J. Persson and P. Ballone, J. Chem. Phys. 112, 9524 (2000).

[16] F. Mugele and M. Salmeron, Phys. Rev. Lett. 84, 5796 (2000).

[17] F. Mugele, S. Baldelli, G.A. Somorjai, and M. Salmeron, J. Phys. Chem. B 104, 3140 (2000).

[18] F. Mugele and M. Salmeron, J. Chem. Phys. (to be published).

[19] D. Kessler, J. Koplik, and H. Levine, Adv. Phys. 37, 255 (1988).

[20] P. Wesseling, An Introduction to Multigrid Methods (Wiley and Sons, New York, 1992).

[21] Numerical Recipes in C: The Art of Scientific Computing (Cambridge University Press, Cambridge, England, 1988). 